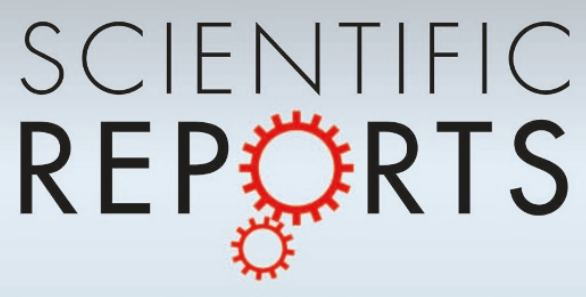

OPEN

SUBJECT AREAS:

TWO-DIMENSIONAL

MATERIALS

SYNTHESIS AND PROCESSING

Received

2 June 2014

Accepted

28 July 2014

Published

26 August 2014

Correspondence and requests for materials should be addressed to T.K. (twkang@sogang. ac.kr)

\footnotetext{
* These authors contributed equally to this work.
}

\section{Two-dimensional Hyper-branched Gold Nanoparticles Synthesized on a Two-dimensional Oil/Water Interface}

\author{
Yonghee Shin ${ }^{1 *}$, Chiwon Lee ${ }^{1 *}$, Myung-Seok Yang ${ }^{2}$, Sunil Jeong' ${ }^{1}$ Dongchul Kim² \& Taewook Kang'
}

\begin{abstract}
'Department of Chemical and Biomolecular Engineering, Sogang University, Seoul, 121-742, Korea, ${ }^{2}$ Department of Mechanical Engineering, Sogang University, Seoul, 121-742, Korea.
\end{abstract}

Two-dimensional (2D) gold nanoparticles can possess novel physical and chemical properties, which will greatly expand the utility of gold nanoparticles in a wide variety of applications ranging from catalysis to biomedicine. However, colloidal synthesis of such particles generally requires sophisticated synthetic techniques to carefully guide anisotropic growth. Here we report that $2 \mathrm{D}$ hyper-branched gold nanoparticles in the lateral size range of about $50 \sim 120 \mathrm{~nm}$ can be synthesized selectively on a $2 \mathrm{D}$ immiscible oil/water interface in a few minutes at room temperature without structure-directing agents. An oleic acid/water interface can provide diffusion-controlled growth conditions, leading to the structural evolution of a smaller gold nucleus to 2D nanodendrimer and nanourchin at the interface. Simulations based on the phase field crystal model match well with experimental observations on the 2D branching of the nucleus, which occurs at the early stage of growth. Branching results in higher surface area and stronger near-field enhancement of $2 \mathrm{D}$ gold nanoparticles. This interfacial synthesis can be scaled up by creating an emulsion and the recovery of oleic acid is also achievable by centrifugation.

T he unique physical and chemical properties of two-dimensional (2D) metallic nanoparticles have attracted significant interest as promising candidates for various applications including catalysis ${ }^{1-2}$, solar-energy harvesting ${ }^{3}$, electronics $s^{4}$, metamaterials $s^{5-7}$ and biomedicine ${ }^{8-10}$. So far, $2 \mathrm{D}$ nanoparticles have been prepared on solid substrates by micro/nanofabrication ${ }^{11-12}$. Their applications have, however, been limited by the number of particles and the need for a solid substrate. Colloidal synthesis of these particles requires delicate control over reaction conditions such as chemicals, reaction time and temperature ${ }^{13-20}$. Therefore, colloidal synthesis typically proceeds either via temperature control, over a long reaction time, through multiple reaction steps, or with the aid of structure-directing agents such as surfactants or a sacrificial template, which often makes reproduction and scaling up difficult. In particular, branched structures such as metallic nanodendrimers, have been considered difficult to produce and only relatively large dendrimers $(>200 \mathrm{~nm}$ ) have been synthesized with the aid of a sacrificial graphene oxide ${ }^{20}$.

Interestingly, here we demonstrate that two-dimensional immiscible oil/water interfaces can provide diffusion-controlled growth conditions, leading to the fast formation of $2 \mathrm{D}$ hyper-branched gold nanoparticles at room temperature without structure-directing agents.

A small nucleus grows on an oleic acid/water interface into a nanodendrimer, and further structural evolution from dendrimer to urchin takes place while the thicknesses of these nanoparticles remains nearly unchanged, confirming that the nanoparticles on the interface are 2D (Fig. 1a). The morphological transition to nanourchin is completed within a few minutes. The experimental data for the $2 \mathrm{D}$ branching of the nucleus at the early stage of growth is consistent with simulations based on the phase field crystal model.

In addition to the aforementioned advantage of having a fast reaction time, no requirement for structural directing agents and room temperature synthesis, the interfacial synthesis of $2 \mathrm{D}$ gold nanoparticles has more practical implications (Fig. 1b). The synthesis can be scaled up by creating an emulsion, allowing the production of a large amount of colloidal particles in a short time. Oleic acid is biocompatible and can be easily recovered due to the different densities of oil (i.e., oleic acid) and water after the interfacial synthesis. 2D hyper-branched gold nanoparticles can show a large surface area that is available to neighboring molecules without diffusion limitation, strong near-field enhancement and tunable far-field response. The size can be as small as $c a$. $50 \mathrm{~nm}$, which is well suited for in vivo applications. 


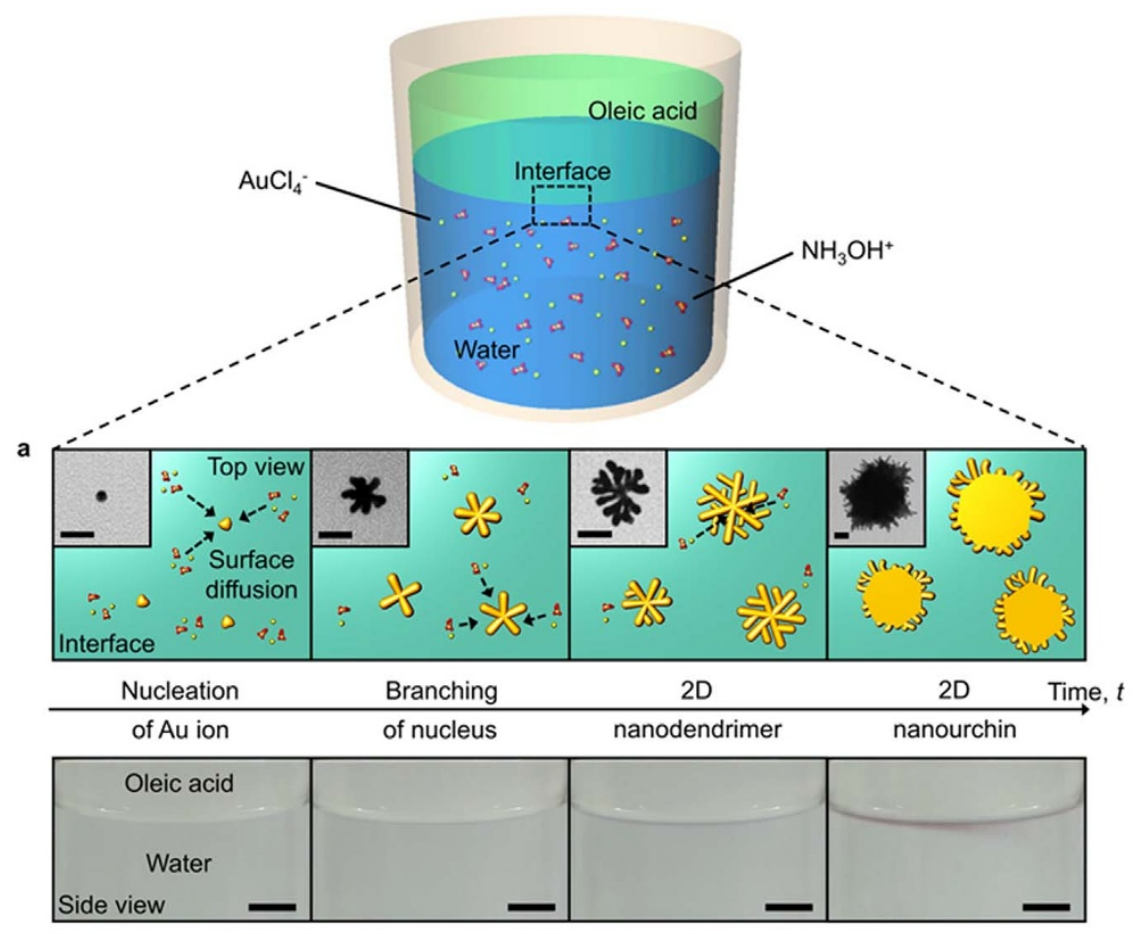

b

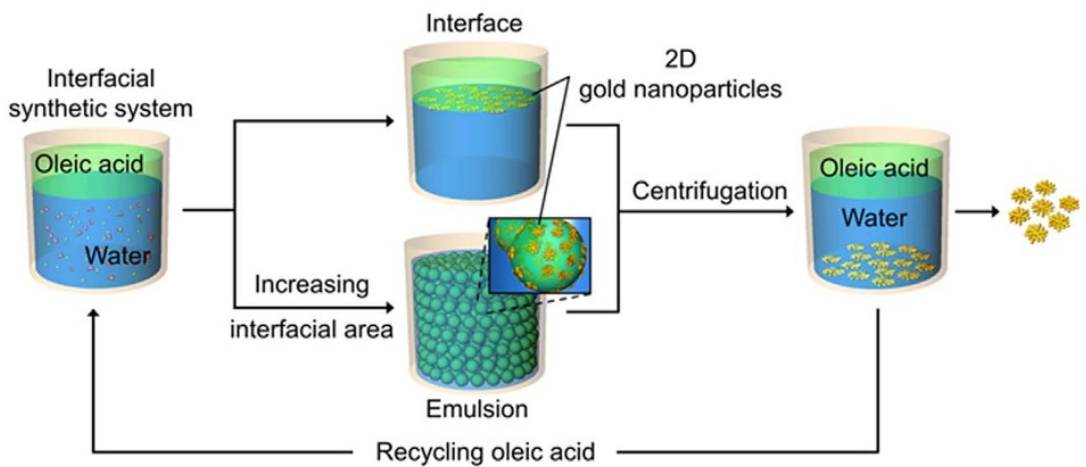

Figure 1 Schematic illustrations and an experimental demonstration for our oil/water interfacial synthesis of two-dimensional (2D) gold nanoparticles. (a), Schematic representation and time-resolved optical images of an oleic acid/water interface for the synthesis of 2D gold nanoparticles (b), Schematic illustration of the interfacial synthesis for recycling of the biocompatible oleic acid and scaling up (creating an emulsion). Scale bars are 25 $\mathrm{nm}$ (transmission electron microscopy (TEM) images in (a)) and $5 \mathrm{~mm}$ (optical images in (a)), respectively.

\section{Results}

Liquid/liquid interfacial synthesis of sub-100 $\mathrm{nm}$ two-dimensional hyper-branched gold nanoparticles. Schematic illustrations and an experimental demonstration for our liquid/liquid interfacial synthesis of two-dimensional (2D) gold nanoparticles are presented in Fig. 1 and Supplementary Movie 1, respectively. Briefly, a gold precursor $\left(\mathrm{HAuCl}_{4} \cdot 3 \mathrm{H}_{2} \mathrm{O}\right.$, tetrachloroauric acid trihydrate) was dissolved in deionized water, followed by the addition of a reducing agent $\left(\mathrm{NH}_{2} \mathrm{OH} \cdot \mathrm{HCl}\right.$, hydroxylamine hydrochloride) into the aqueous solution. Oleic acid was then slowly poured on top of the solution. Approximately $4 \mathrm{~min}$ after the addition of the oleic acid, the liquid/ liquid interface appeared light grey in color and gradually changed to pink (Fig. 1a). On the other hand, both water and oil layers remained colorless for over $30 \mathrm{~min}$, indicating that the formation of the nanoparticles selectively takes place at the interface.

In order to characterize the gold nanoparticles formed on the interface in detail, the oleic acid/water interface was sampled for transmission electron microscope (TEM) and atomic force microscope (AFM) measurements. To statistically analyze their morphological and structural transitions, 80 particles from each microscope image were randomly selected (Supplementary Fig. 1 and Supple- mentary Fig. 2). Fig. 2a shows representative TEM and AFM images of the gold nanoparticles collected from the interface at the early stage of growth (at $4 \mathrm{~min}$ after the addition of oleic acid). Clearly, the gold nanoparticles show a dendritic morphology along with primary and secondary branches (Fig. 2a and Supplementary Fig. 1). The TEM images show that small gaps in the range of $2-8 \mathrm{~nm}$ exist between branches. Average lengths with respect to $x, y$, and $z$ axes of the particles were $53.5 \pm 10.4 \mathrm{~nm}, 54.2 \pm 11.7 \mathrm{~nm}$, and 4.21 $\pm 1.70 \mathrm{~nm}$, respectively (Fig. $2 \mathrm{~b}$ and Fig. $2 \mathrm{~g}$ ), indicating that the gold nanoparticles are two-dimensional. Note that the number of secondary branches is dependent on their lateral size (i.e., more secondary branches were observed for larger lateral sizes) while that of primary branches is similar irrespective of the size (Supplementary Fig. 3). The width of both the primary and secondary branches is about $7 \mathrm{~nm}$.

As the reaction time proceeds, the small gaps between the branches are quickly filled with gold atoms and new branches are formed simultaneously (Fig. 2c and Supplementary Fig. 1). After $5 \mathrm{~min}$, the morphology of the particles changed from nanodendrimer to nanourchin with many smaller branches $(4-7 \mathrm{~nm})$ on the outer surface (Fig. 2e and Supplementary Fig. 1). The lengths of the 
a

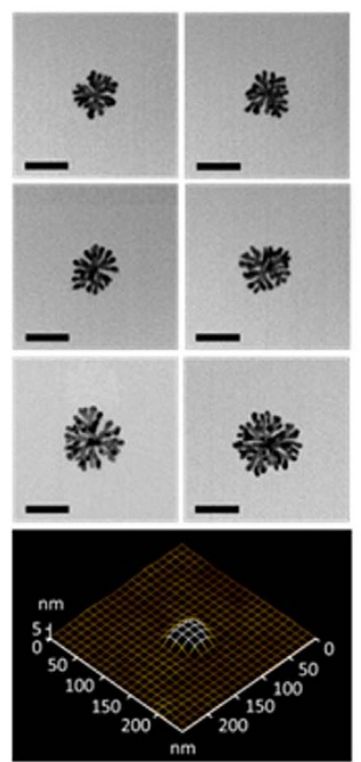

b
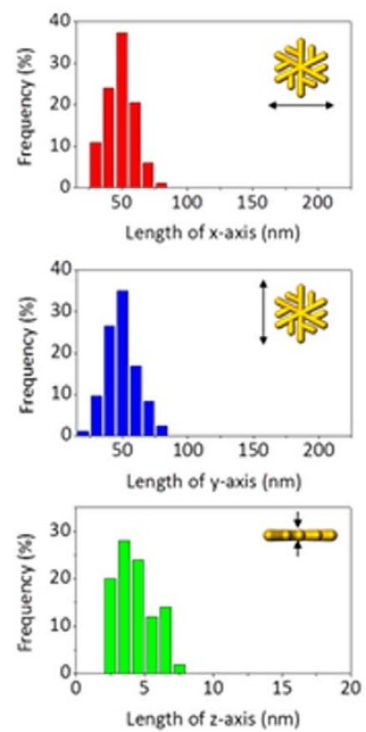

c

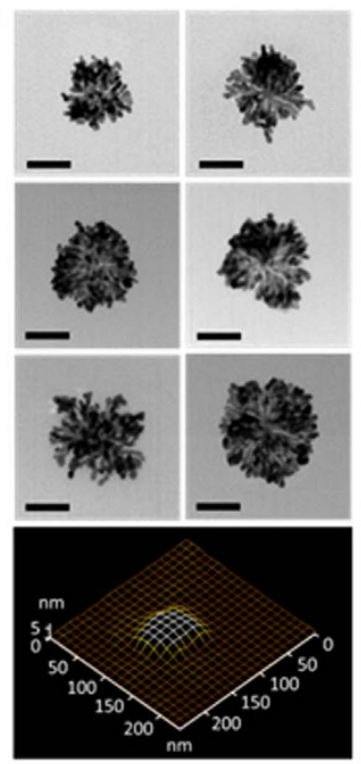

d
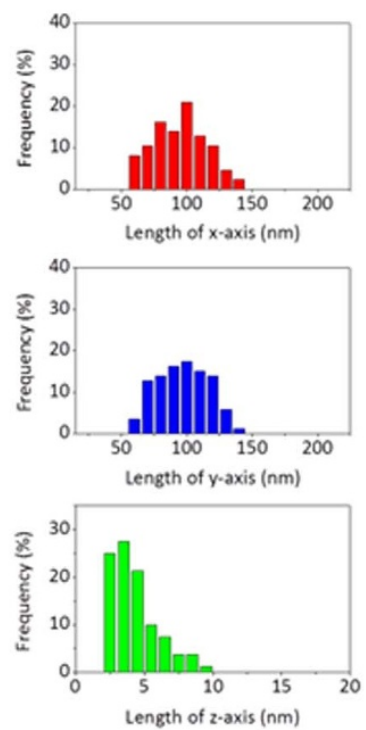
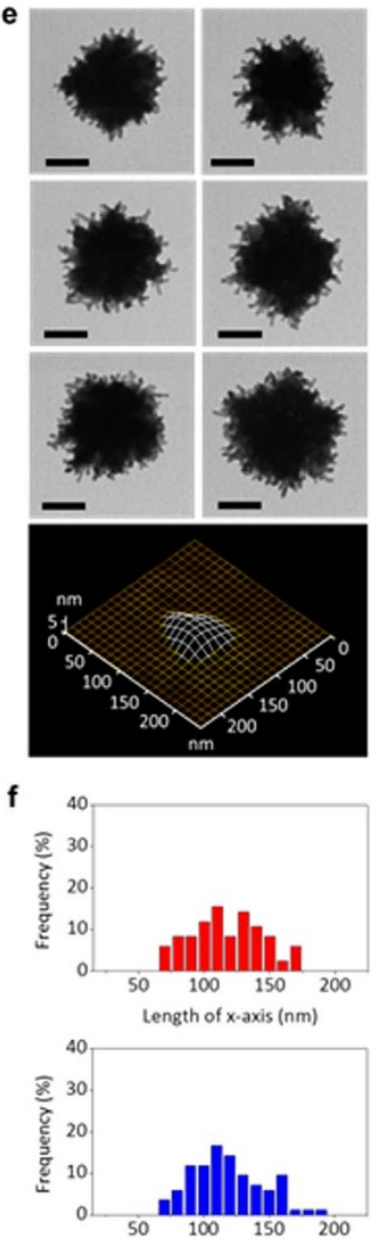

Length of $y$-axis (nm)

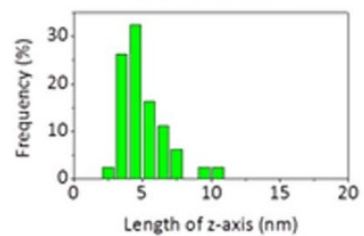

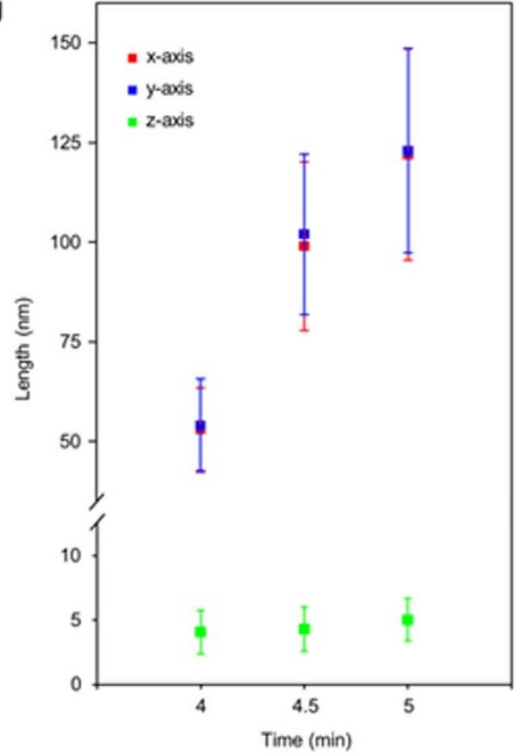

h

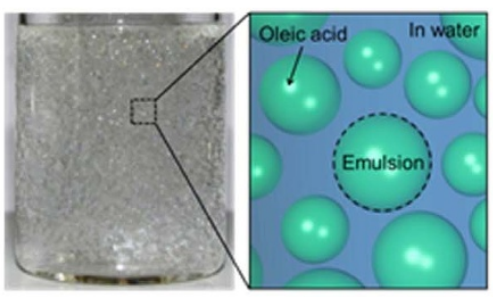

i

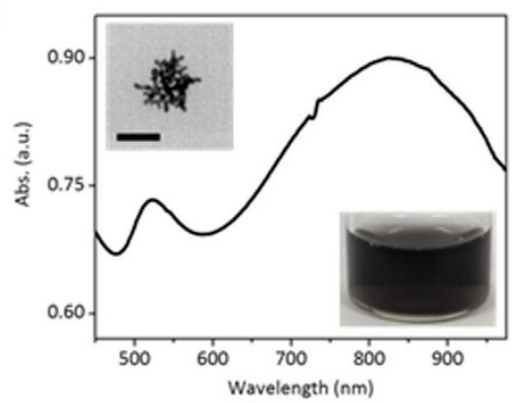

Figure 2 Morphological and optical characterizations of 2D hyper-branched gold nanoparticles. (a), (c), (e), Representative TEM and atomic force microscopy (AFM) images of 2D gold nanoparticles sampled at the interface at (a) 4 min, (c) 4 min $30 \mathrm{~s}$, and (e) 5 min, respectively. (b), (d), (f), Size distributions with respect to x- (red), y- (blue) and z-axes (green) of the particles. (g), Average lengths with respect to x- (red), y- (blue) and z-axis (green) of the particles with increasing reaction time. (h), Optical image and corresponding cartoon of the interfacial synthesis via the oleic acid-in-water emulsion. (i), Representative UV-vis spectrum of the gold nanodendrimer synthesized at the oleic acid-in-water emulsion (top inset: TEM image of the nanoparticle, bottom inset: optical image of the solution). For statistical analyses, 80 particles are randomly selected (Supplementary Fig. 1 and Supplementary Fig. 2). Scale bar is $50 \mathrm{~nm}$.

nanourchin, with respect to $\mathrm{x}, \mathrm{y}$, and $\mathrm{z}$ axes were found to be $122.0 \pm$ $26.5 \mathrm{~nm}, 123.0 \pm 25.7 \mathrm{~nm}$, and $5.03 \pm 1.65 \mathrm{~nm}$, respectively (Fig. $2 \mathrm{f}$ and Fig. 2g). During this morphological transition, lateral sizes with respect to $\mathrm{x}$ and $\mathrm{y}$ axes increased by about $140 \%$ while the thickness (i.e., along $\mathrm{z}$ axis) remained nearly constant. This suggests that the gold nanoparticles formed on the oleic acid/water interface are likely to grow along the interface, maintaining their two-dimensional structure. Note that as the reaction time further increased, the lateral sizes of the nanoparticles increased linearly to over $215 \mathrm{~nm}$ and the branches disappeared simultaneously. The thickness slightly increased to about $17 \mathrm{~nm}$ (Supplementary Fig. 1d).

Since the reducing agent and oleic acid are ionizable depending on solution $\mathrm{pH}$, we examined the effect of solution $\mathrm{pH}$ on the formation of gold nanoparticles on the interface. The reaction batches were prepared separately by varying $\mathrm{pH}$ from 3.05 to 6.28 (Supple- mentary Fig. 7). As solution $\mathrm{pH}$ was further decreased (i.e., more acidic than $\mathrm{pH} 3.36$ ), a color change was not observed at the oleic acid/water interface. At higher $\mathrm{pH}$, the color change was observed not only at the interface but also in solution. The gold nanoparticles synthesized at higher $\mathrm{pH}$ were no longer two-dimensional. Based on these experimental observations, the interfacial reaction mechanism can be estimated as follows. As gold precursor $\left(\mathrm{HAuCl}_{4}\right)$ dissolves in water, the $\mathrm{pH}$ is decreased to 3.36. The reducing agent, hydroxylamine $\left(\mathrm{NH}_{2} \mathrm{OH}\right)$ is likely to be protonated to $\mathrm{NH}_{3} \mathrm{OH}^{+}$since the $\mathrm{pK}_{\mathrm{a}}$ of $\mathrm{NH}_{2} \mathrm{OH}$ is around 5.9. Due to the weaker reducing capability of $\mathrm{NH}_{3} \mathrm{OH}^{+}$compared to $\mathrm{NH}_{2} \mathrm{OH}$, the reduction of the $\mathrm{Au}$ ion is strongly limited in solution. However, at the oleic acid/water interface, the carboxylic head group of the oleic acid would assist in the reduction of $\mathrm{Au}$ ions, allowing both the formation of a small gold nucleus at the interface ${ }^{21-22}$ and the growth along the interface. 

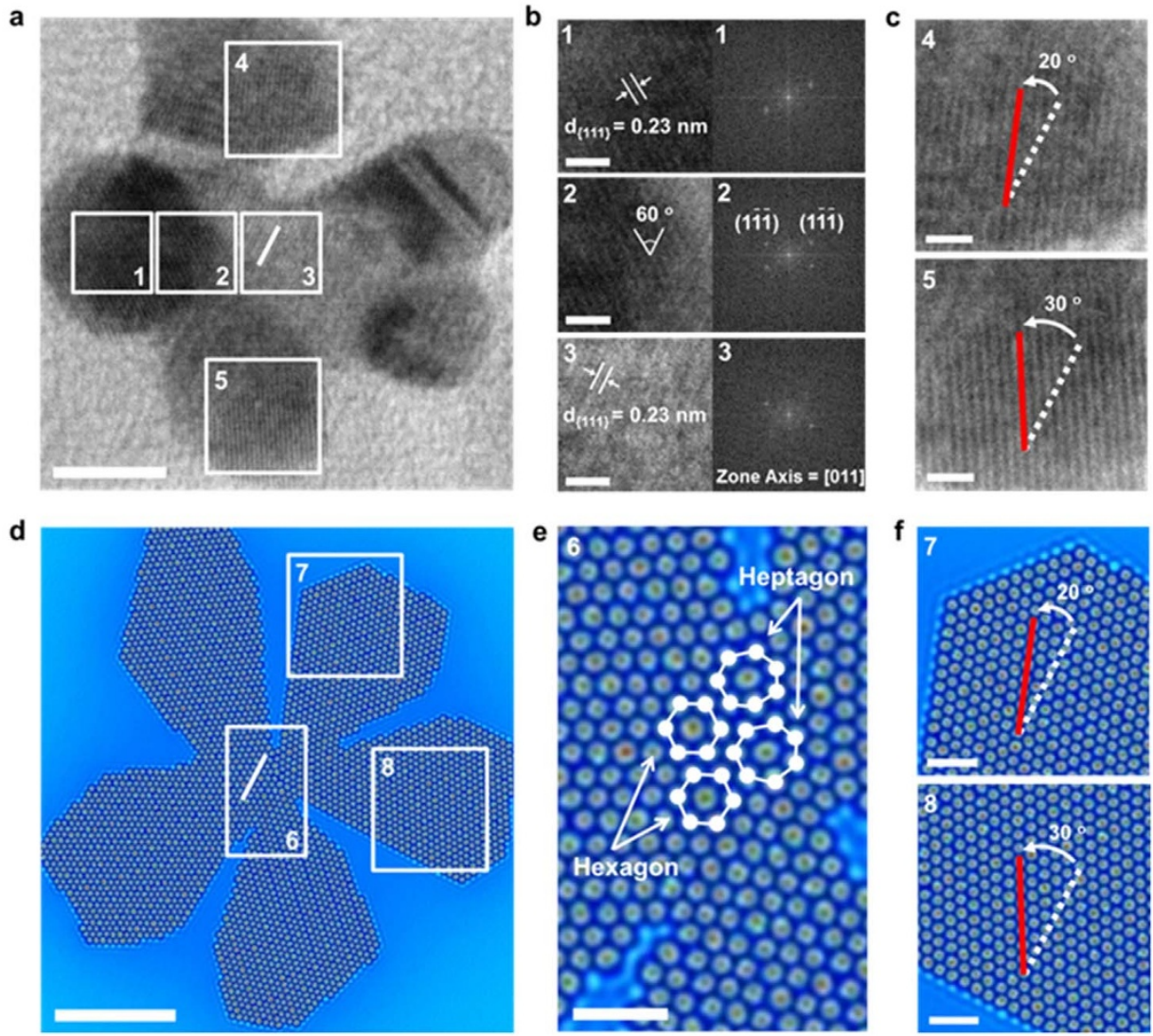

Figure 3 Crystallographic analysis and phase field crystal simulation of 2D gold nanoparticles. (a), High-resolution TEM image of 2D nanodendrimer obtained at the early stage of growth. Crystal lattice of the core (marked with white line) is set as a reference to compare the orientation.

(b), Lattice-resolved images and electron diffraction patterns taken along the [011] zone axis from the core to branches (numbered 1-3 boxes in (a)). (c), Magnified images (numbered 4,5 boxes in (a)) showing tilted crystal lattice orientations between the core (white dotted line) and each branch (red line). (d), Phase field crystal model to simulate the early stage of growth. (e), The appearance of non-hexagonal packing of surface Au atoms between the core and branch (numbered 6 box in (d)). (f), Magnified simulation images (numbered 7,8 boxes in (d)) for tilted crystal lattice orientations. Scale bars are $5 \mathrm{~nm}(\mathrm{a}, \mathrm{d})$ and $1 \mathrm{~nm}(\mathrm{~b}, \mathrm{c}, \mathrm{e}, \mathrm{f})$, respectively.

Scaling up the interfacial synthesis by creating an emulsion. Even though our 2D gold nanoparticles undergo a structural transition from nanodendrimer to nanouchin, the reaction intermediate, for example, a gold nanodendrimer can also be selectively obtained by either increasing the interfacial area or decreasing the concentration of the gold precursor. The oleic acid-in-water emulsion was produced by stirring the solution at a moderate speed (Fig. $2 \mathrm{~h}$ and see Methods section for the detailed preparation method). The stirring speed dictates the size of the emulsion. The gold nanoparticles were collected by centrifugation and re-dispersed in water. As shown in Fig. $2 \mathrm{i}$, the final solution is dark grey in color. The absorbance spectrum of the solution exhibits two distinct surface plasmon resonance (SPR) bands at 525 and $828 \mathrm{~nm}$ (Fig. 2i). Two SPR bands in the visible and near-IR range can be attributed to the shape anisotropy (i.e., two-dimensional structure) of the particles $^{23-25}$. TEM images of the particles clearly show the dendritic morphology with primary and secondary branches, which is the same as that of the particles obtained on the planar oleic acid/ water interface (Top inset of Fig. $2 \mathrm{i}$ and Supplementary Fig. 8). In addition, the thicknesses of these particles were measured by AFM. The average thickness is found to be $7.03 \pm 1.12 \mathrm{~nm}$, indicating that the particles obtained from the oil-in-water emulsion are 2D (Supplementary Fig. 9). Note that the selectivity of the nanodendrimer over unwanted by-products such as gold spherical nanoparticles is also dependent on the stirring speed.

The crystallographic analysis and phase field crystal simulation of 2D gold nanodendrimers. Fig. 3a shows representative high- resolution TEM (HRTEM) images of 2D gold nanodendrimers obtained at the early stage of growth. Lattice characteristics of (111) planes were observed from both the core and a branch of the particle (Fig. 3b and Supplementary Fig. 10). Interestingly, tilted crystal lattice orientations between the core and each branch were observed (Fig. 3c). The phase field crystal simulation also produces the branching and tilted crystal lattice orientations which match well with the experimental observation (Fig. 3d, Fig. 3f and Supplementary Movie 2). Furthermore, the crystal growth simulation results suggest that the tilted crystal orientation angles would result from the appearance of non-hexagonal packing of Au atoms (e.g., heptagonal packing) (Fig. 3e).

Electromagnetic field simulation and Surface-enhanced Raman spectroscopy (SERS) measurements. Next, electromagnetic field (EM) simulation was carried out using the finite-difference timedomain (FDTD) method (see Supplementary Methods for the detailed simulation). The EM simulation results for three kinds of 2D gold nanoparticles (i.e., 2D gold nanoparticles with primary and secondary branches, with primary branches, and without any branches) are shown in Fig. $4 \mathrm{a}-\mathrm{c}$. The number of areas for strong EM enhancement (hot spot) increases with branching (Fig. 4d). Strong EM enhancement is generated in small gaps between branches.

In order to realize the benefit of our 2D hyper-branched gold nanoparticles, 2D gold nanodendrimers, as a proof-of-concept, were tested for surface-enhanced Raman spectroscopy (SERS). 4-chlorobenzenethiol (CBT) was selected as a standard molecule for SERS. 
a

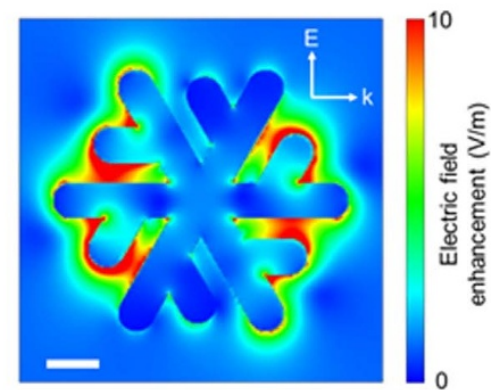

b

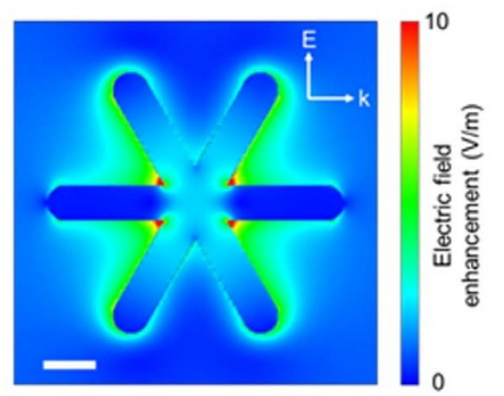

C

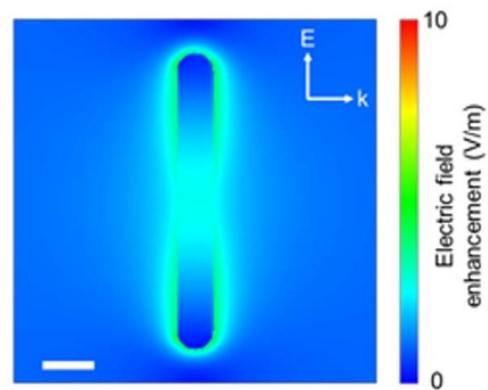

d

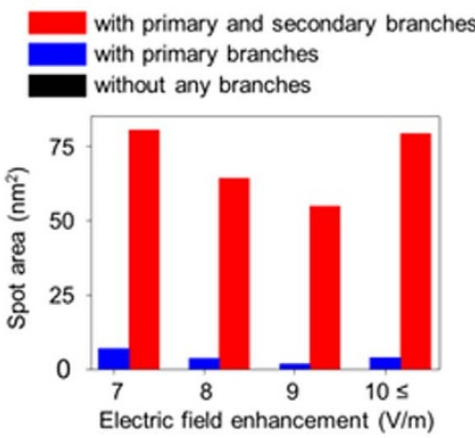

e

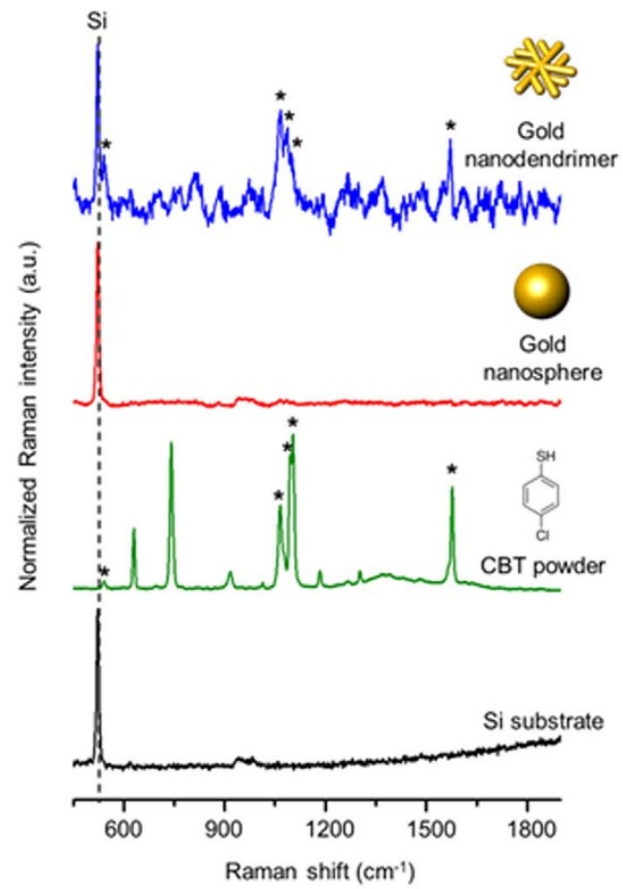

Figure 4 | Electromagnetic (EM) simulation of 2D gold nanoparticles and surface-enhanced Raman spectroscopy (SERS) experiment with the 2D gold nanodendrimer. $(\mathrm{a}-\mathrm{c})$, Electric field enhancement for 2D gold nanoparticles with (a) primary and secondary branches, (b) primary branches only, without (c) any branches. Scale bar corresponds to $10 \mathrm{~nm}$. (d), Plot of hot spot area of 2D gold nanoparticles with the enhancement factor larger than 7 $(\mathrm{V} / \mathrm{m})$. (e), SERS spectra when using colloidal gold nanodendrimer solution (blue line) and $20 \mathrm{~nm}$ gold nanospheres solution (red line). Raman spectra of 4-chlorobenzenethiol (CBT) powder (green line) and silicon substrate (black line). The Raman transitions of CBT are marked with asterisks (542, 1066, 1086,1102 , and $1571 \mathrm{~cm}^{-1}$, respectively).

For the control experiment, gold nanospheres (GNS), $20 \mathrm{~nm}$ in diameter (Supplementary Fig. 12) were tested to compare the SERS signal under the experimental conditions since GNS has a similar amount of $\mathrm{Au}$ atoms to the gold nanodendrimer (Supplementary Table 1). From ICP-MS analysis, the concentrations of each nanoparticle were measured to be the same (Supplementary Table 1). Fig. 4e shows normalized SERS spectra of CBT from a colloidal gold nanodendrimer and GNS solutions. No apparent Raman transition is found in the Raman spectrum taken from colloidal GNS (red line in Fig. 4e). In contrast, characteristic Raman transitions of CBT (assigned by asterisks, blue line in Fig. 4e) are clearly observed from colloidal gold nanodendrimer solutions. This SERS enhancement from gold nanodendrimers can be attributed to many factors including a large accessible surface area without diffusion limitation, many hot spots between branches, and the wavelength of the laser. Several studies are currently underway to address each effect in detail but are beyond the scope of this study.

\section{Discussion}

In conclusion, we have demonstrated interfacial synthesis that lead to the fast formation of two-dimensional gold nanoparticles at room temperature without structure-directing agents. The small gold nucleus with a diameter of about $4 \mathrm{~nm}$ selectively grows on the interface into a nanodendrimer due to branching at an early stage of growth, which is consistent with numerical simulations based on the phase field crystal model. Gold nanodendrimers further undergo the transition to a larger gold nanourchin as the reaction time proceeds. During this structural transformation, interestingly, the thickness of these nanoparticles is maintained at around $5 \mathrm{~nm}$, indicating that they are two dimensional. The synthesis method described in this paper does not require structural directing agents, has a fast reaction time, consumes less energy, uses a biocompatible and recyclable solvent and is scalable for production. From a material standpoint, we expect that these 2D gold nanoparticles have a substantial impact on catalysis, biomedicine and other areas of research due to their high surface area without diffusion limitation and high packing density, tunable size and excellent near and far-field optical properties.

\section{Methods}

Synthesis of two-dimensional (2D) gold nanoparticles at an oleic acid/water interface. Two-dimensional gold nanoparticles were synthesized in a $30 \mathrm{ml}$ glass vial. $0.850 \mathrm{ml}$ hydrogen tetrachloroaurate(III) hydrate solution $\left(\mathrm{HAuCl}_{4} \cdot 3 \mathrm{H}_{2} \mathrm{O}, 1.2 \mathrm{mg}\right.$ / $\mathrm{ml}$ ) was mixed with $12.8 \mathrm{ml} \mathrm{DI}$ water, and $37.5 \mu \mathrm{l}$ of aqueous hydroxylamine hydrochloride $\left(\mathrm{NH}_{2} \mathrm{OH} \cdot \mathrm{HCl}, 0.05 \mathrm{M}\right)$ was added to the solution. After homogeneous mixing, $2.8 \mathrm{ml}$ of oleic acid was slowly introduced to form the oleic acid-water interface. Within a few minutes, color was observed at the interface after the addition of oleic acid. $1 \mathrm{ml}$ of the aqueous phase just below the oleic acid-water interface was collected using a pipette at $4 \mathrm{~min}, 4 \mathrm{~min} 30 \mathrm{~s}, 5 \mathrm{~min}, 6 \mathrm{~min}$ and $7 \mathrm{~min}$.

Synthesis of 2D gold nanoparticles in oleic acid-in-water emulsions. $1.53 \mathrm{ml}$ of hydrogen tetrachloroaurate(III) hydrate solution $\left(\mathrm{HAuCl}_{4} \cdot 3 \mathrm{H}_{2} \mathrm{O}, 0.715 \mathrm{mg} / \mathrm{ml}\right)$ was diluted with $162.27 \mathrm{ml}$ DI water in a 1-neck round-bottom flask, followed by the addition of $450 \mu \mathrm{l}$ of hydroxylamine hydrochloride aqueous solution $\left(\mathrm{NH}_{2} \mathrm{OH} \cdot \mathrm{HCl}\right.$, $0.05 \mathrm{M}$ ) with stirring. Under vigorous stirring, $33.6 \mathrm{ml}$ of oleic acid was quickly added to the solution to form oleic acid-in-water emulsions. The reaction in the emulsion mixture was allowed to proceed for $20 \mathrm{~min}$ while it was stirred continuously with a magnetic stirrer. After $30 \mathrm{~s}$ the stirring was stopped and the mixture was separated into water and oleic acid, and $150 \mathrm{ml}$ of the aqueous phase was collected. The synthesized nanoparticles in the aqueous solution were isolated by centrifugation (5000 rpm, $10 \mathrm{~min}$ ) and subsequently re-dispersed in water. $20 \mu \mathrm{l}$ of colloidal gold nanodendrimer solution was dropped onto a carbon-coated 300 mesh TEM grid (Inc. Ted Pella) and the UV-VIS extinction spectra were taken on a JASCO V530 spectrophotometer.

Growth mechanism simulation via the phase field crystal model. We used a phase field crystal model to simulate the growth of a $2 \mathrm{D}$ gold nanodendrimer ${ }^{26-29}$. This model relies on the dimensionless density, $\varphi=\left(\rho-\rho_{r e f}\right) / \rho_{\text {ref }}$, where $\rho$ and $\rho_{\text {ref }}$ are the time averaged particle density and a reference solution of particle density, respectively. The detailed explanation for the growth mechanism simulation is included in the Supplementary Methods.

Particle preparation and functionalization for SERS measurements. For SERS measurements, the gold nanodendrimer and gold nanosphere were functionalized 
with a $10 \mathrm{mM}$ ethanolic solution of 4-chlorobenzenethiol for $3 \mathrm{~h}$ under magnetic stirring at room temperature. Functionalized particles were re-dispersed in water after being centrifuged three times $(8000 \mathrm{rpm}, 10 \mathrm{~min})$ in order to eliminate the remaining ethanol and 4-chlorobenzenethiol. The concentrations of functionalized gold nanodendrimer and gold nanospheres in solution, obtained by ICP-MS, were converted to the number per unit volume for each sample.

Raman and SERS measurements. A Raman spectrometer QE65000 from Ocean Optics Inc. and $785 \mathrm{~nm}$ laser module I0785MM0350MS from Innovative Photonic Solution Inc. were used for Raman and SERS measurements. Raman measurements were carried out for 4-chlorobenzenethiol powders, $10 \mathrm{mM}$ ethanolic solution of 4chlorobenzenethiol, ethanol solutions, and the silicon substrate. The Raman measurement was conducted using a $785 \mathrm{~nm}$ laser at a power of $250 \mathrm{~mW}$ and an integration time of $10 \mathrm{~s}$. SERS measurements were conducted with $50 \mu \mathrm{l}$ of functionalized gold nanodendrimers and $50 \mu \mathrm{l}$ of functionalized gold nanospheres on a silicon substrate. The SERS measurement also used a $785 \mathrm{~nm}$ laser at a power of $250 \mathrm{~mW}$ and an integration time of $10 \mathrm{~s}$. The baseline of the SERS spectrum was corrected before normalization.

1. Huang, X. et al. Freestanding palladium nanosheets with plasmonic and catalytic properties. Nat. Nanotech. 6, 28-32 (2011).

2. Larsson, E. M., Langhammer, C., Zoric, I. \& Kasemo, B. Nanoplasmonic Probes of Catalytic Reactions. Science 326, 1091-1094 (2009).

3. Kulkarni, A. P. et al. Plasmon-Enhanced Charge Carrier Generation in Organic Photovoltaic Films Using Silver Nanoprisms. Nano Lett. 10, 1501-1505 (2010).

4. Moon, G. D. et al. Highly Stretchable Patterned Gold Electrodes Made of $\mathrm{Au}$ Nanosheets. Adv. Mater. 25, 2707-2712 (2013).

5. Shegai, T. et al. A bimetallic nanoantenna for directional colour routing. Nat. Commun. 2, 481 (2011).

6. Larouche, S. et al. Infrared metamaterial phase holograms. Nat. Mater. 11 450-454 (2012).

7. Liu, N. et al. Infrared Perfect Absorber and Its Application As Plasmonic Sensor. Nano Lett. 10, 2342-2348 (2010).

8. Homan, K. A. et al. Silver Nanoplate Contrast Agents for in Vivo Molecular Photoacoustic Imaging. ACS Nano 6, 641-650 (2012).

9. Jones, M. R. et al. Plasmonically Controlled Nucleic Acid Dehybridization with Gold Nanoprisms. Chem. Phys. Chem 10, 1461-1465 (2009).

10. Ye, E. et al. Plasmonic Gold Nanocrosses with Multidirectional Excitation and Strong Photothermal Effect. J. Am. Chem. Soc. 133, 8506-8509 (2011).

11. Koh, A. L. et al. High-Resolution Mapping of Electron-Beam-Excited Plasmon Modes in Lithographically Defined Gold Nanostructures. Nano Lett. 11 1323-1330 (2011).

12. Fredriksson, H. et al. Hole-Mask Colloidal Lithography. Adv. Mater. 19, 4297-4302 (2007).

13. Chen, S. \& Carrol, D. L. Synthesis and Characterization of Truncated Triangular Silver Nanoplates. Nano Lett. 2, 1003-1007 (2002).

14. Xiong, Y. et al. Kinetically Controlled Synthesis of Triangular and Hexagonal Nanoplates of Palladium and Their SPR/SERS Properties. J. Am. Chem. Soc. 127, 17118-17127 (2005)

15. Zhang, Q. et al. Seeded Growth of Uniform Ag Nanoplates with High Aspect Ratio and Widely Tunable Surface Plasmon Bands. Nano Lett. 10, 5037-5042 (2010).

16. Xiong, Y. \& Xia, Y. Shape-Controlled Synthesis of Metal Nanostructures: The Case of Palladium. Adv. Mater. 19, 3385-3391 (2007).

17. Millstone, J. E. et al. Observation of a Quadrupole Plasmon Mode for a Colloidal Solution of Gold Nanoprisms. J. Am. Chem. Soc. 127, 5312-5313 (2005).

18. Millstone, J. E., Metraux, G. S. \& Mirkin, C. A. Controlling the Edge Length of Gold Nanoprisms via a Seed-Mediated Approach. Adv. Funct. Mater. 16, 1209-1214 (2006)
19. Huang, X. et al. Synthesis of hexagonal close-packed gold nanostructures. Nat. Commun. 2, 292 (2011)

20. Jasuja, K. \& Berry, V. Implantation and Growth of Dendritic Gold Nanostructures on Graphene Derivatives: Electrical Property Tailoring and Raman Enhancement. ACS Nano 3, 2358-2366 (2009).

21. Goncalves, G. et al. Surface Modification of Graphene Nanosheets with Gold Nanoparticles: The Role of Oxygen Moieties at Graphene Surface on Gold Nucleation and Growth. Chem. Mater. 21, 4796-4802 (2009).

22. Zan, R., Bangert, U., Ramasse, Q. \& Novoselov, S. Evolution of Gold Nanostrucures on Graphene. Small 7, 2868-2872 (2011).

23. Jana, N. R., Gearheart, L. \& Murphy, C. J. Seed-mediated growth approach for shape-controlled synthesis of spheroidal and rod-like gold nanoparticles using a surfactant template. Adv. Mater. 13, 1389-1393 (2001).

24. Ah, C. S. et al. Size-Controlled Synthesis of Machinable Single Crystalline Gold Nanoplates. Chem. Mater. 17, 5558-5561 (2005).

25. Jin, R. et al. Photoinduced conversion of silver nanospheres to nanoprisms. Science 294, 1901-1903 (2001).

26. Swift, J. \& Hohenberg, P. C. Hydrodynamic fluctuations at the convective instability. Phys Rev. A 15, 319-328 (1977).

27. Ramakrishnan, T. V. \& Yussouff, M. First-principles order-parameter theory of freezing. Phys Rev. B 19, 2775-2794 (1979).

28. Elder, K. R. \& Grant, M. Modeling elastic and plastic deformations in nonequilibrium processing using phase field crystals. Phys Rev. E 70, 051605 (2004).

29. Tegze, G., Toth, G. I. \& Granasy, L. Faceting and Branching in 2D Crystal Growth. Phys Rev Lett. 106, 195502 (2011).

\section{Acknowledgments}

This research was supported by the International Research \& Development Program of the National Research Foundation of Korea (NRF) funded by the Ministry of Science, ICT \& Future Planning (MSIP) (No. NRF-2013K1A3A1A32035444) and the Basic Science Research Program through the NRF funded by the MSIP (No. NRF-2013R1A1A2011263).

\section{Author contributions}

T.K. conceived this concept. Y.S., C.L. and T.K. designed the experiments. Y.S., C.L. and S.J. performed the experiments. M.Y and D.K. contributed to simulations. Y.S., C.L. and T.K. wrote the manuscript. All authors discussed the results and commented on the manuscript.

\section{Additional information}

Supplementary information accompanies this paper at http://www.nature.com/ scientificreports

Competing financial interests: The authors declare no competing financial interests.

How to cite this article: Shin, Y. et al. Two-dimensional Hyper-branched Gold Nanoparticles Synthesized on a Two-dimensional Oil/Water Interface. Sci. Rep. 4, 6119; DOI:10.1038/srep06119 (2014)

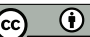

This work is licensed under a Creative Commons Attribution 4.0 International License. The images or other third party material in this article are included in the article's Creative Commons license, unless indicated otherwise in the credit line; if the material is not included under the Creative Commons license, users will need to obtain permission from the license holder in order to reproduce the material. To view a copy of this license, visit http://creativecommons.org/licenses/by/4.0/ 